\title{
LIBERTAD DE EXPRESIÓN Y LÍMITES DEMOCRÁTICOS
} FREEDOM OF SPEECH AND DEMOCRATIC CONSTRAINT

\section{José Luis Castro-Montero*}

Resumen: La literatura jurídica ha teorizado ampliamente las razones por las cuales la libertad de expresión es un derecho fundamental: a nivel individual, constituye una condición necesaria para la autonomía y, a nivel público, promueve la fiscalización de las autoridades y el descubrimiento de la verdad a través del flujo de información en el "mercado de ideas". En este ensayo se presenta una visión alternativa e integradora: la libertad de expresión como fundamento y condición de la legitimidad política y la igualdad democrática. Esta premisa resulta importante para entender la libertad de expresión y sus limitaciones. Paradójicamente, ciertas restricciones a la libertad de expresión, especialmente aquellas que aluden a la difamación de autoridades públicas, pueden afectar gravemente la propia legitimidad de las leyes antidiscriminación. En este sentido, la aplicación de las normas antidiscriminación en favor de autoridades públicas produce el efecto adverso al deseado ya que se excluye a voces disidentes del debate público. Contrariamente, cuando las ideas se expresan en público, la legitimidad de las leyes antidiscriminación se fortalece. Para explicar el efecto adverso de la limitación a la libertad de expresión, este ensayo explora la relación entre la opinión pública, la legitimidad política y la igualdad democrática. Particularmente, el caso ecuatoriano sirve de ejemplo para ilustrar estas tesis.

Palabras clave: Democracia, libertad de opinión, legitimidad política, restricciones, Ecuador

Abstract: There is a significant literature justifying why freedom of speech should be seen as a fundamental right. At the individual level, freedom of speech should

\footnotetext{
* Abogado por la Pontificia Universidad Católica del Ecuador. Estudios en Derecho Constitucional en la Universidad Andina Simón Bolívar (Quito, Ecuador). LL.M. (c) por la Universidad de Tilburg (Países Bajos) y la Universidad Católica de Lovaina (Bélgica). j.1.castromontero@tilburguniversity.edu
} 
be protected to further autonomy. In the public domain, freedom of speech is not only essential for the discovery of truth through the marketplace of ideas, but also fosters citizens' checks over public officials. The present essay takes an alternative but at the same time integrative approach, by stating that freedom of speech is a necessary condition of political legitimacy and democratic equality. This is particularly important to understand the limits of freedom of speech, as certain laws forbidding free speech may also annihilate political legitimacy to enforce non-discriminatory regulation. In this regard, special laws that protect public officials defamation produce an unintended effect over dissident voices, as these laws exclude them from public debates. Conversely, legitimacy of non-discriminatory laws is justified when opinions are publicly expressed. Advancing this unintended effect of non-discriminatory regulation enforcement, this essay explores the close interdependency of public opinion, political legitimacy and democratic equality. Particularly the Ecuadorian case serves well to illustrate this viewpoint.

Keywords: Democracy, Free Speech, Political Legitimacy, Limitations, Ecuador

Sumario. I. Introducción. II. La libertad de expresión como derecho fundamental. III. La libertad de expresión y la legitimidad política. IV. La libertad de expresión como presupuesto democrático. V. Las restricciones al libre discurso público. VI. La limitación a la libertad de expresión en Ecuador. VII. Conclusión y perspectivas. Referencias.

\section{INTRODUCCIÓN}

Como es sabido, la libertad puede ser entendida desde dos categorías. Desde una perspectiva negativa, la libertad implica la ausencia de barreras u obstáculos. Positivamente, en cambio, la libertad se traduce en la autodeterminación y la capacidad de un agente para controlar su propio destino (Berlín, 1969). En particular, la libertad de expresión es uno de los valores fundamentales de las democracias constitucionales que conjuga estas dos categorías. A diferencia de la libertad de pensamiento, no existe libertad de expresión incondicional o absoluta, pero a su vez las restricciones a esta libertad no deben constituir obstáculos desproporcionados.

Tres ideas justifican la libertad de expresión como derecho fundamental: a nivel individual, constituye una condición de posibilidad de la autonomía, mientras que a nivel público, promueve la fiscalización de las autoridades y el descubrimiento de la verdad a través del flujo de información en el "mercado de ideas". En este ensayo se presenta una visión alternativa e integradora: la libertad de expresión como fundamento de la legitimidad política en regímenes democráticos. Dworkin (2009), por ejemplo, ha destacado el valor de la participación en pie de igualdad, dado 
que en una democracia justa y legítimamente justificable se reconoce que cada ciudadano cuenta con un voto, pero también con la oportunidad de expresar su voz ${ }^{1}$. De acuerdo con esta visión, la mayoría no debe obstaculizar la expresión del individuo. Caso contrario, se anula el debate democrático y se invalida la única justificación democrática de la mayoría para imponer normas, especialmente, aquellas indeseadas por la minoría.

Lo dicho resulta importante si se considera que ciertas restricciones a la libertad de expresión pueden afectar la legitimidad de las leyes antidiscriminación. Precisamente, la hipótesis que se defiende en este ensayo sostiene que la regulación irracional a la libertad de expresión debilita la justificación de las normas que promueven la no discriminación. Este efecto indeseado de las normas que promueven la no discriminación pone en tela de juicio la idoneidad de ciertas restricciones a la libertad de expresión, como por ejemplo la difamación de autoridades públicas.

El presente ensayo se encuentra articulado en seis apartados. Primero, se describen varias teorías atinentes a la justificación de la libertad de expresión como derecho fundamental. Luego, se analiza la vinculación de esta libertad con la legitimidad política. En el tercer apartado se conceptualiza la libertad de expresión como condición necesaria para el juego democrático. En el cuarto apartado se discuten los posibles efectos adversos de las restricciones a la expresión. En particular, se concluye que ciertas restricciones, como la difamación a las autoridades públicas, afectan la participación en pie de igualdad de las personas y anulan la legitimidad democrática de las leyes antidiscriminación. Finalmente, se presenta un caso sucedido en el Ecuador que ilustra la aplicación de estos argumentos teóricos.

\section{LA LIBERTAD DE EXPRESIÓN COMO DERECHO FUNDAMENTAL}

De modo sucinto, se puede clasificar las teorías que justifican la libertad de expresión como derecho fundamental en tres categorías:

\footnotetext{
${ }^{1}$ Dworkin (2009) sostiene:
}

«La decisión de la mayoría no es justa a menos que cada uno haya tenido la oportunidad de expresar sus opiniones, gustos, presunciones, prejuicios o ideales, no solamente esperando influenciar a los demás, pero también para confirmar su posición de agente interesado, en lugar de víctima pasiva, de la acción colectiva. La mayoría no puede imponer su voluntad a quien ha prohibido el derecho de expresar su opinión, ya sea que este haya elegido protestar, objetar o argumentar frente a la decisión mayoritaria, antes que una decisión sea tomada» (p. V). 
autonómicas, filosófico-económicas y políticas ${ }^{2}$. Las corrientes autonómicas resaltan el valor intrínseco de la libertad de expresión en cuanto la misma resulta un elemento esencial para el desarrollo de la personalidad y la autonomía (Colburn, 2010). La autonomía, sin embargo, es una categoría muy amplia que puede ser relacionada esencialmente con cualquier libertad $^{3}$. Las principales críticas a estas escuelas sostienen que la autonomía no es un fundamento exclusivo de la libertad de expresión y que la protección de la autonomía de algunas personas podría evitar el desarrollo de la personalidad de otras (Bork, 1971).

Las corrientes filosófico-económicas tienen su origen en el pensamiento de John Milton (1897), para quien la competencia de ideas favorece al descubrimiento de la verdad, y de John Stuart Mill (2003), quien mantiene que la protección de la libertad de expresión promueve la capacidad racional de los individuos para discriminar la verdad de la mentira. De acuerdo a estas corrientes, la metáfora del mercado de las ideas ilustra el valor instrumental e intrínseco de la libertad de expresión. Instrumentalmente, la expresión permite que las ideas puedan ser formuladas y debatidas en público. El debate (o como explica Milton, la competencia) permite la difusión de información sobre un fenómeno. El valor intrínseco de la expresión, en cambio, se evidencia a medida que mientras más ideas se expresan, los individuos cuentan con mayor capacidad racional para participar en debates futuros.

Varias críticas se han planteado frente a las corrientes filosóficoeconómicas. Entre ellas, el acceso al mercado de las ideas puede desembocar en un problema de exclusión y, como sucede en el mercado económico, los más poderosos podrían controlar la oferta y demanda (Tribe, 1978). Otra fuerte crítica a esta metáfora la ha esgrimido

\footnotetext{
${ }^{2}$ La libertad de expresión ha sido consagrada en el art. 19 de la Declaración Universal de los Derechos Humanos y formulaciones similares se han reproducido en los arts. 19 y 20 del Pacto Internacional de Derechos Civiles Políticos, el art. 13 de la Convención Americana de Derechos Humanos, el art. 10 de la Convención Europea de Derechos Humanos y la mayoría de constituciones. Cualquiera, entonces, bien podría afirmar que la libertad de expresión es un derecho fundamental porque a nivel nacional e internacional ha sido reconocida como tal. A pesar de la veracidad de esta afirmación, también es cierto que la justificación de un derecho requiere un entendimiento más amplio sobre su contenido, funcionalidad y límites.

${ }^{3}$ Sobre la vaguedad del concepto de autonomía Gerald Dworkin (1988) ha señalado:

«El concepto de "autonomía" ha sido usado (...) en ocasiones como equivalente de libertad (...) otras como equivalente de auto gobierno o soberanía, otras como un concepto ligado a la voluntad. También es equivalente a la dignidad, a la integridad, a la individualidad, a la independencia, a la responsabilidad, y al autoconocimiento» (p. 6).
} 
Wellington (1979), quien señala que el fin último del mercado de las ideas no es la verdad; las ideas verdaderas no necesariamente prevalecen sobre las falsas y los individuos generalmente no actúan como entes racionales capaces de eliminar la mentira.

Las corrientes políticas justifican la libertad de expresión como catalizador del escrutinio a las autoridades públicas, enfatizando la estrecha relación de la expresión y la democracia. En este sentido, la libertad de expresión es necesaria para mantener a los electores informados sobre las actuaciones de los gobernantes. Más importante aún, esta libertad permite a los ciudadanos condicionar las actuaciones de los gobernantes (Meiklejohn, 1961). Blasi (1977), por ejemplo, indica que la libertad de expresión actúa como medio de fiscalización en regímenes democráticos. El problema de esta corriente radica en su aplicación exclusiva al ámbito público.

En este punto conviene notar que ninguna de estas teorías se acepta de modo absoluto y universal. Tampoco son mutuamente exclusivas, sino más bien complementarias en cuanto a su contenido argumentativo. Vale también advertir el énfasis de cada una de las corrientes sobre el valor intrínseco e instrumental de la libertad de expresión.

A continuación, se presenta una perspectiva complementaria e integrativa para justificar la libertad de expresión como derecho fundamental. De modo general, se sostiene que la libertad de expresión es una condición necesaria para la participación en pie de igualdad y, por ello, constituye un fundamento de legitimidad política en regímenes democráticos. Esta premisa es importante para entender el alcance de ciertas restricciones a la libertad de expresión.

\section{LA LIBERTAD DE EXPRESIÓN Y LA LEGITIMIDAD POLÍTICA}

La legitimidad política alude principalmente a las condiciones para gobernar e imponer normas de modo coercitivo (Scharpf, 2009). El concepto de legitimidad política puede abarcarse desde una perspectiva descriptiva y otra normativa. Descriptivamente, la legitimidad se manifiesta cuando los ciudadanos creen que una entidad política los gobierna y, en consecuencia, obedecen las obligaciones a ellos impuestas (Weber, 2009). Normativamente, la legitimidad política se refiere a los criterios que vuelven a un orden político digno de respeto y de obediencia (Habermas, 2015). Como hace notar Scharpf (2009), las «creencias socialmente compartidas sobre la legitimidad crean una obligación 
normativa que ayuda a asegurar la obediencia voluntaria incluso frente a normas o decisiones gubernamentales no deseadas» (p. 5).

Sobre la base de esta distinción del arraigo de las autoridades políticas, en esta sección se examinan dos cuestiones: primero, la obligación, entendida desde el ámbito descriptivo, de obedecer leyes que promueven la no discriminación que tienen aquellos individuos coartados de emitir opiniones y, luego, la legitimidad normativa para exigir el cumplimiento de normas que limitan la libertad de opinión, especialmente de aquellos cuyos derechos políticos han sido circunscritos por las limitaciones de las leyes que promueven la no discriminación.

La relación entre la participación en pie de igualdad y la legitimidad política ha sido ampliamente analizada desde la Ilustración. La primera vinculación entre estos dos conceptos establece que todos los sujetos tienen el mismo valor moral, lo que determina que el Estado debe tratar con igual respeto los intereses de todos los individuos (Kant, 2000). De ahí que para resolver el dilema del disentimiento sobre la aplicación de normas coercitivas frente a ciudadanos libres, se propone el juego democrático como aquel sistema de elección colectiva que proporciona la legitimidad más defendible para establecer un orden social (Habermas, 2015).

Conviene notar que la igualdad de oportunidades es una condición de posibilidad de la legitimidad política en regímenes democráticos. Como explica Dahl (1985), el juego democrático supone que los ciudadanos puedan participar como iguales cuando se establecen normas imperativas que regulan la vida de cada uno de los miembros de una comunidad. La participación en pie de igualdad, en consecuencia, permite a los ciudadanos reivindicar sus intereses subjetivos y expresar su sentir sobre el interés colectivo. Politólogos muestran, sobre la base de la evidencia empírica, que la participación en el debate y la toma de decisiones fomenta el compromiso y la obediencia de los ciudadanos frente al derecho (Tyler, 2000).

Desde una perspectiva normativa, la aplicación de una norma que prohíbe el discurso de alguien que no participó en el debate para instituir dicha norma, ni que tampoco está de acuerdo con su contenido, viola el principio fundamental de participación en pie de igualdad. Esta prohibición irrespeta la condición del individuo como entidad moral digna de respeto. En efecto, la participación en pie de igualdad resulta necesaria para establecer cualquier condición normativa de legitimidad política.

Ahora bien, si al igual que Dahl (1985) se argumenta que la participación en pie de igualdad es una condición de posibilidad de la democracia, se debe también reconocer que las normas sobre las cuales cabe el disentimiento de los ciudadanos se vuelven ilegítimas cuando se 
niega la participación de los miembros de la comunidad política en el debate político. En contraposición con aquellas normas que consagran los mandatos básicos de cualquier sociedad, cuando la legitimidad de una norma cuya calidad es cuestionable no puede ser razonablemente discutida, se anula la legitimidad política.

\section{LA LIBERTAD DE EXPRESIÓN COMO PRESUPUESTO DEMOCRÁTICO}

A pesar de la estrecha vinculación de la libertad de expresión con otros derechos políticos, usualmente no se percibe la magnitud que las limitaciones a la libertad de expresión pueden tener sobre la igualdad y la legitimidad democrática. La prohibición no razonable del derecho al voto, por ejemplo, limitaría gravemente cualquier decisión en un régimen democrático. No es para nada difícil imaginar las graves consecuencias que dicha prohibición acarrearía para la legitimidad política. Sin embargo, tiende a mirarse con mayor contemplación las restricciones a la libertad de expresión.

A continuación se analiza cómo las restricciones a la libertad de expresión pueden anular la legitimidad democrática, debido principalmente a su estrecha vinculación con la opinión pública.

Hace más de cincuenta años, Hans Kelsen (1961) explicó:

La voluntad de la comunidad, en una democracia, siempre se produce a través de una discusión de mayoría y minoría, de la libre consideración de argumentos a favor y en contra de una regulación. Esa discusión no solamente tiene lugar en el parlamento, sino también, y ante todo, en reuniones políticas, en los periódicos, libros y otros medios de opinión pública. Una democracia sin opinión pública no es más que una contradicción semántica (pp. 287-288).

Como se mencionó en la primera sección del presente ensayo, es propiamente a través de la opinión pública que la gente controla a sus representantes públicos (Jacoby, 2000). Su opinión y la voluntad de expresarla como pública, conlleva la oportunidad de manifestar su sentimiento individual, pero también de inmiscuirse en la organización de la sociedad (Schamis et al., 2002; Sunstein, 1995). Las restricciones a la libertad de expresión a nivel público, por ello, impiden que las personas cuenten con suficiente información para realizar elecciones conscientes (Posner, 1986). Consecuentemente, la limitación irracional de la libertad de 
expresión no solamente afecta la autonomía y los intereses de los individuos, pero además afecta la igualdad y legitimidad democrática ${ }^{4}$.

A partir de aquello, se concluye que las restricciones a la opinión pública afectan la participación en pie de igualdad, circunstancia que a su vez acarrea graves consecuencias para la legitimidad del sistema democrático. A partir de este razonamiento, a continuación se expone cómo las restricciones a la libre opinión pueden comprometer la justificación de las leyes que promueven la no discriminación, en términos de legitimidad democrática.

\section{LAS RESTRICCIONES AL LIBRE DISCURSO PÚBLICO}

En este punto, debe notarse que el propósito de este ensayo no es poner en tela de juicio la validez de restricciones a la libertad de expresión como la privacidad o el discurso de odio. Como habrá advertido el lector atento, más bien se pretende evaluar la validez de la difamación a las autoridades públicas como límite al libre discurso público.

Como los teóricos del mercado de las ideas, Waldron (2012) señala que las restricciones a la libertad de expresión están permitidas en tanto que no afecten el acceso a la información para la posterior toma decisiones colectivas. Dicha afirmación no puede defenderse como un universal, según lo sostenido en este ensayo.

En primer lugar, como se expuso, las prohibiciones a la libertad de opinión afectan directamente el auto gobierno democrático y la participación en pie de igualdad. Una ley antidiscriminación puede, en efecto, coartar la opinión de un individuo y debilitar la justificación de su aplicación de modo coercitivo (Nie, Powell \& Prewitt, 1969). En este sentido, se produce el resultado adverso al deseado, ya que se discrimina a quienes se prohíbe emitir su opinión y se debilita la justificación de la aplicación forzosa de la ley que promueve la no discriminación. Además, el efecto es doblemente dañino para quienes disienten con el contenido de la norma antidiscriminación y no pueden expresarlo. Primero, porque su discurso ha sido coartado y, segundo, porque se les obliga a respetar una norma con la que no están de acuerdo.

${ }^{4}$ Sobre este punto, Weinsten (2011) sostiene:

«Si un individuo es excluido de participar en un discurso público porque el gobierno disiente con su punto de vista (...) cualquier decisión que resulte de tal discusión, carece de legitimidad, sobre todo si se aplica frente al individuo excluido» (p. 468). 
Por otra parte, no se trata de defender a ultranza la opinión independientemente de su contenido, porque de ningún modo resultaría justificable que un sujeto $\mathrm{X}$ denigre la condición humana de otro sujeto $\mathrm{Y}$ (Posner, 1986; Richardst, 1970). Al contrario, se trata de establecer el balance adecuado para que a $X$ se le permita emitir su opinión, sin antes haberle prohibido su discurso, pero además, se trata de exigirle a $\mathrm{X}$ el cumplimiento de una norma, una vez que haya podido expresarse. Entonces cabe preguntarse si la selección impositiva de normas que limitan la libertad de expresión, sobre la base de la difamación a las autoridades públicas, pueden ser justificadas.

En contextos democráticos, ciertas limitaciones a la libertad de expresión no deberían aplicarse a priori, sin considerar su costo para el debate democrático y la legitimidad política. Principalmente porque, como decía Dworkin (2009), la libertad de opinión confirma la posición de las personas como agentes interesados, «en lugar de víctima pasiva, de la acción colectiva» (p. V). Por ello, no debe subestimarse la afectación que las limitaciones a la libertad de expresión pueden acarrear para la legitimidad e igualdad política.

La sobrerregulación del derecho a la libertad de expresión, por los argumentos previamente expuestos, conlleva un efecto doblemente pernicioso. Por una parte afecta la participación en pie de igualdad de las personas ya que las desconoce como individuos morales y, por otra, anula la legitimidad democrática de las leyes antidiscriminación ${ }^{5}$.

\section{LA LIMITACIÓN A LA LIBERTAD DE EXPRESIÓN EN ECUADOR}

En el Ecuador, el gobierno ha propugnado el activismo estatal para promocionar leyes antidiscriminación, que regulan los denominados derechos de participación y comunicación. Como destaca Ramos (2013, p.

\footnotetext{
${ }^{5}$ De aquello se deriva la peligrosidad de interpretaciones extensivas de las limitaciones a la libertad de expresión que, por ejemplo, ha realizado la Corte Constitucional del Ecuador en la Sentencia 047-15-SIN-CC, de septiembre de 2015, mediante la cual se señaló:

«Conforme se desprende de la jurisprudencia citada, el derecho al honor y buen nombre constituye un limitante a la libertad de expresión en cualquiera de sus formas, ya que este último no puede ser ejercido de tal manera que afecte negativamente otros derechos constitucionales; por tanto, la libertad de expresión no es un derecho absoluto y debe ser interpretado en su integralidad con los demás derechos establecidos en el texto constitucional con el objetivo de que (sic) este sea ejercido de manera adecuada sin afectar la honra de terceras personas» (p. 16).
} 
67), la aparente «democratización de la comunicación mediatizada (...) alerta sobre las acciones gubernamentales que han resultado desdemocratizadoras». En particular entidades como el Consejo de Regulación y Desarrollo de la Información y Comunicación (CORDICOM) y la Superintendencia de la Información y Comunicación (SUPERCOM) han adquirido protagonismo luego de reiteradas sanciones administrativas a medios de comunicación masiva y a periodistas.

Vivimos en (un) Estado de Derecho, nadie está por encima de ley, los medios deben ser responsables del contenido que publican y la gente responsable por la difusión de contenidos discriminatorios por razón de sexo y orientación sexual (Carlos Ochoa, Superintendente de Información y Comunicación, 2015) ${ }^{6}$.

Sobre la base de este tipo de discurso, las autoridades que encabezan dichas entidades justifican prácticas antidemocráticas que ilustran los argumentos teóricos que aquí se sostienen.

De acuerdo al Informe de Rendición de Cuentas de la SuPERCOM (2015), se han iniciado 438 procedimientos administrativos sancionatorios a nivel nacional. De aquellos, 175 fueron propuestos directamente por ciudadanos y 263 fueron iniciado de oficio por la SUPERCOM. En total, 299 casos concluyeron con una resolución sancionatoria ${ }^{7}$. La mayoría de resoluciones aluden a la norma antidiscriminatoria establecida en el art. 62 de la Ley Orgánica de Comunicación, que establece:

Art. 62. «Prohibición.- Está prohibida la difusión a través de todo medio de comunicación social de contenidos discriminatorios que tenga por objeto o resultado menoscabar o anular el reconocimiento, goce o ejercicio de los derechos humanos reconocidos en la Constitución y en los instrumentos internacionales. Se prohíbe también la difusión de mensajes a través de los medios de comunicación que constituyan apología de la discriminación e incitación a la realización de prácticas o actos violentos basados en algún tipo de mensaje discriminatorio».

Quizás uno de los casos más notorios y pertinentes para el presente análisis es la sanción al caricaturista Xavier Bonilla (Bonil), expedida por la SUPERCOM en 2015. En una de sus caricaturas publicadas en el diario $E l$ Universo, Bonil incluyó dos fotos del exfutbolista de la selección ecuatoriana de ascendencia afroecuatoriana, que actualmente desempeña el cargo de asambleísta nacional por el partido Alianza País, Agustín "El Tin" Delgado, en el que se podía leer, por un lado «Con mi discurso todos me

\footnotetext{
${ }^{6}$ Cfr. Bonil (2015): «Donde yo veo un asambleísta, ellos ven a un negro o un afro».

${ }^{7}$ Cfr. Más de 300 ciudadanos reciben informe de Rendición de Cuentas de la Supercom (SUPERCOM, 26-III-2015).
} 
dicen pobre Tin» y por otro «pero con mi sueldo de asambleísta ya nadie me dice pobretón» (cfr. Bonil, 8-II-2015).

Bonil fue sancionado por haber discriminado con su caricatura al asambleísta Delgado por su condición socio-económica y racial. Asimismo, la CORDICOM, mediante un informe emitido el 23 de diciembre de 2014, concluyó que la caricatura de Bonil incluía «discriminación indirecta mediante la cual se hace distinción respecto de la actividad política y laboral del asambleísta Agustín Delgado, miembro del colectivo afroecuatoriano en razón de su condición socioeconómica». «Donde yo veo un asambleísta, ellos ven a un negro o un afro» (cfr. Bonil, 8-II-2015), replicó Bonil. La resolución emitida por la SUPERCOM además previno al caricaturista y al diario para que en el futuro puedan «corregir y mejorar sus prácticas para el pleno y eficaz ejercicio de los derechos a la comunicación». Además se les conminó «a abstenerse de reincidir en el cometimiento de actos que se encuentran reñidos con la Ley Orgánica de Comunicación».

Más allá del gran revuelo mediático que alcanzó dicho procedimiento administrativo y que Bonil había sido sancionado previamente por el contenido de otra de sus caricaturas en 2014 (mediante Resolución 001DNGJPO-INPS de la SUPERCOM), este caso refleja la problemática aplicación de leyes antidiscriminación que establecen restricciones al derecho a la libertad de expresión. Particularmente se evidencia cómo ciertas restricciones a la libertad de expresión se aplican de modo ilegítimo y antidemocrático en un contexto de legalismo politizado (Conaghan, 2015).

Primero conviene notar el modo a través del cual sanciones de orden administrativo eliminaron la posibilidad debatir sobre las funciones oficiales de Delgado como asambleísta y representante de la ciudadanía ecuatoriana. Lejos de crear una cultura de respeto a la diversidad y al pluralismo, restricciones antidiscriminatorias sirvieron de excusa para invalidar cualquier tipo de discusión racional y democrática sobre los argumentos expuestos por Delgado en su discurso. En una comunidad democrática, la exposición del disenso frente a normas que restringen el derecho a la libertad de expresión no debería justificar la restricción de opiniones de quienes se oponen al contenido de dichas normas y mucho menos de quienes fiscalizan el quehacer de las autoridades públicas a través de medios de opinión masivos (Johnston, 1997). Caso contrario, la propia norma antidiscriminación se vuelve ilegítima, debido a que contraría su propio propósito: evitar la discriminación y la exclusión.

Ahora bien, en un régimen democrático las autoridades públicas deben tratar con igual respeto los intereses de todos los ciudadanos. Mayorías o minorías, oposición y oficialismo, las opiniones disidentes 
deben ser públicamente expresadas. Caso contrario, la coerción sobre los disidentes resulta inmoral e injustificable jurídicamente (Fiss, 1985).

En el caso del caricaturista Bonil, se incumplen dos presupuestos básicos de la legitimidad política debido a que, primero, se obliga a un individuo a quien se le ha limitado su derecho a la libertad de expresión a obedecer una norma que promueve la no discriminación y, segundo, se exige el cumplimiento de medidas que limitan la libertad de opinión contra quien no puede participar como un igual para reivindicar sus intereses subjetivos y expresar su sentir sobre el interés colectivo. ¿Cómo entonces las entidades gubernamentales exigen el cumplimiento del derecho en contra de los ciudadanos libres y autónomos quienes razonablemente disienten con la ley? La respuesta, a esta inquietud desgraciadamente, no puede extraerse del contenido conceptual de la democracia, sino de regímenes de tinte autoritario.

Continuando con el análisis, como se afirmó previamente, la participación en el debate y la toma de decisiones fomenta el compromiso y la obediencia de las normas por parte de los ciudadanos, incluso cuando los mismos no están de acuerdo con el contenido de ciertas normas. Desde esta perspectiva, no se puede negar la oportunidad de modo selectivo al caricaturista, periodista, médico, albañil, ciudadano... que desea exponer su criterio razonado. De lo contrario, se irrespeta la condición del individuo como entidad moral digna de respeto.

Para concluir, vale evidenciar que la aplicación de una norma que prohíbe el discurso, en este contexto, irrespeta el principio fundamental de igualdad democrática. Principalmente, porque no se puede exigir el cumplimiento de una norma a un sujeto a quien se prohibió participar en el debate para instituir dicha norma, negándole a su vez la opinión sobre el contenido de la misma. En consecuencia, de acuerdo al argumento sostenido, cualquier decisión sancionatoria en el caso analizado carece de legitimidad política.

\section{CONCLUSIÓN Y PERSPECTIVAS}

De modo extensivo y profundo la literatura jurídica ha teorizado las razones por las cuales la libertad de expresión es un derecho fundamental. Tres ideas centrales subyacen a los discursos que sustentan dicha caracterización. Primero, se destaca la estrecha relación entre democracia y libertad de expresión, debido al flujo de ideas que cumple una doble función. Por una parte, se informa a los electores con el objeto de optimizar la calidad de las decisiones individuales y, por otra, se promueve 
la fiscalización de las autoridades públicas. Segundo, se alude a la libertad de expresión como fuente fundamental del "mercado" de las ideas, metáfora que alude al acceso de los individuos a recursos que permiten el intercambio de conocimientos y saberes, y el descubrimiento de la verdad. Por último, se alude a la libertad de expresión como condición necesaria para el desarrollo de la autonomía individual.

Este ensayo adopta una visión integradora y a la vez alternativa - la libertad de expresión como fundamento de legitimidad política en regímenes democráticos - para sostener que la restricción de la libertad de expresión no justifica, por sí misma, la exclusión del debate público de quienes disienten con los estándares establecidos por las normas que promueven la no discriminación. Al contrario, si se restringe la libertad de expresión, se afecta la legitimidad política de las normas antidiscriminación, tanto en un sentido descriptivo como normativo, especialmente en el caso de quienes disienten con el contenido de aquellas normas.

Ya en la práctica, el efecto adverso de ciertas sanciones administrativas que defienden la no discriminación mediante la restricción de la libertad de expresión resulta paradójico, dado que carecen de legitimidad para ser aplicadas dentro de un sistema democrático, sobre todo frente a quienes disienten con el contenido de aquellas decisiones. En este sentido, es menester de las autoridades públicas y en general de los estudiosos de las políticas públicas, diseñar mecanismos que regulen la libertad de expresión sin limitarla drásticamente. De lo contrario, lo que se destruye - junto con las libertades individuales - es la legitimidad política de un régimen democrático.

La libertad de expresión como fundamento de la legitimidad política en regímenes democráticos abre las posibilidades para el diseño de una agenda de investigación tanto teórica como empírica sobre el desarrollo de esta libertad no solo a nivel nacional, sino también regional. Futuras contribuciones académicas encontrarán terrenos inexplorados en el análisis de la libertad de expresión en ámbitos privados, particularmente relacionados con la era tecnológica y el internet. Esto debido a que de ningún modo la justificación de la expresión puede restringirse al ámbito político o público. Quizás las contribuciones más importantes también impliquen acercamientos interdisciplinarios, que exploren la vinculación de la libertad de conciencia, la libertad de pensamiento y la autonomía individual como fundamento cultural de la libertad de expresión. Otros campos poco estudiados son los efectos de las nuevas tecnologías desarrolladas por la neurociencia que pretenden manipular el cerebro humano y, en consecuencia, la libertad de expresión. 


\section{REFERENCIAS}

Bayón, J.C. (2000). Democracia y derechos: problemas de fundamentación del constitucionalismo. Plan nacional de investigación científica, desarrollo e innovación tecnológica, financiado por el Ministerio de Ciencia y Tecnología. En https://www.upf.edu/filosofiadeldret/_pdf/bayon-democracia.pdf (recuperado el 9-I-2017).

Berlin, I. (1958). Two concepts of liberty. Oxford: Clarendon Press.

Blasi, V. (1977). The Checking Value in First Amendment Theory. Law \& Social Inquiry, 2(3), 521-649.

Bonil (8-II-2015). Donde yo veo un asambleísta, ellos ven a un negro o un afro. Diario El Universo. En http://www.eluniverso.com/noticias/2015/02/08/nota/4529421/ donde-yo-veo-asambleista-ellos-ven-negro-o-afro (recuperado el 9-I-2017).

Bork, R.H. (1971). Neutral Principles and some First Amendment Problems. Ind. Lj, 47, 1.

Colburn, B. (2010). Autonomy and Liberalism. New York: Routledge.

Conaghan, C. (2015). Surveil and Sanction: The Return of the State and Societal Regulation in Ecuador. ERLACS, (98), 7. doi: 10.18352/erlacs.9979

Dworkin, G. (1988). The Theory and Practice of Autonomy. Cambridge: Cambridge University Press.

Dworkin, R. (2009). A Foreword to Extreme speech and Democracy. Oxford: Oxford University Press.

Dahl, R. (1985). Controlling nuclear weapons. Syracuse, N.Y.: Syracuse University Press.

Fiss, O. M. (1985). Free Speech and Social Structure. Iowa Law Review, 71. Recuperado desde http://heinonline.org/HOL/Page?handle=hein.journals/ilr71\&id=1417\&di $\mathrm{v}=\&$ collection (recuperado el 9-I-2017).

Habermas, J. (1979). Communication and the Evolution of Society. Boston: Beacon Press.

Nie, N.H., Powell, G.B. \& Prewitt, K. (1969). Social Structure and Political Participation: Developmental Relationships, II. American Political Science Review, 63(03), 808-832. doi: 10.1017/S0003055400258607

Jacoby, W. G. (2000). Issue Framing and Public Opinion on Government Spending. American Journal of Political Science, 44(4), 750. doi: 10.2307/2669279

Johnston, R. (1997). Who Deliberates? Mass Media in Modern Democracy Benjamin I. Page Chicago: University of Chicago Press, 1996, pp. ix, 167. Canadian Journal of Political Science, 30(02), 385. doi: 10.1017/S0008423900015675

Kant, I. (2000). The Metaphysics of Morals. Political Writings. Recuperado desde http://www.inp.uw.edu.pl/mdsie/Political_Thought/Kant\%20-\%20groundwork $\% 20$ for\%20the\%20metaphysics\%20of\%20morals\%20with\%20essays.pdf (recuperado el 9-I-2017).

Kelsen, H. (1961). General Theory of Law and State. New York: Russell \& Russell. 
Meiklejohn, A. (1961). The First Amendment is an absolute. The Supreme Court Review, 1961, 245-266.

Mill, J. S. (2003). On liberty. New Heaven: Yale University Press.

Milton, J. (1897). Areopagitica. London: Kegan Paul, Trench, Trübner \& Co.

Peter, F. (2007). Democratic legitimacy and proceduralist social epistemology. Politics, Philosophy \&Amp; Economics, 6(3), 329-353. doi: 10.1177/1470594x07081303

Posner, R. A. (1986). Free Speech in an Economic Perspective. Suffolk University Law Review, 20. En http://heinonline.org/HOL/Page?handle=hein.journals/sufflr20 \&id=31\&div=\&collection= (recuperado el 8-I-2017).

Ramos, I. (2013). Trayectorias de democratización y desdemocratización de la comunicación en Ecuador. Íconos - Revista de Ciencias Sociales, O(46), 67.

Richardst, D. A. J. (1970). Free Speech and Obscenity Law: Toward a Moral Theory of the First Amendment. University of Pennsylvania Law Review, 122(45).

Schamis, H. E., Díaz-Bonilla, E., Schamis, H. E., Frieden, J., Stein, E., Schamis, H.E... Balza, M. (2002). Argentina: Crisis and Democratic Consolidation. Journal of Democracy, 13(2), 81-94. doi: 10.1353/jod.2002.0030

Scharpf, F. W. (2009). Legitimacy in the multilevel European polity. European Political Science Review, 1(02), 173-204.

Sunstein, C. (1995). Democracy and the problem of free speech. Publishing Research Quarterly, 11(4), 58-72. doi: 10.1007/BF02680544

Superintendencia de la Información y Comunicación (26-III-2015). Más de 300 ciudadanos reciben informe de Rendición de Cuentas de la Supercom. En el sitio oficial de la institución: http://www.supercom.gob.ec/es/sala-deprensa/noticias/22-mas-de-300-ciudadanos-reciben-informe-de-rendicion-decuentas-de-la-supercom (recuperado el 9-I-2017).

Superintendencia de la Información y Comunicación (31-I-2014). Resolución 001DNGJPO-INPS. En http://www.eluniverso.com/sites/default/files/archivos/ 2014/01/resolucion_tramite_002_xavier_bonillauniverso_31-01-2014.pdf (recuperado el 9-I-2017).

Tribe, L. H. (1978). Toward a Metatheory of Free Speech. Sw. UL Rev., 10, 237.

Tyler, T. R. (2000). Multiculturalism and the Willingness of Citizens to Defer to Law and to Legal Authorities. Law and Social Inquiry, 25(4), 983-1019.

Waldron, J. (2012). The Harm in Hate Speech. Cambridge: Harvard University Press.

Weber, M. (2009). The theory of social and economic organization. En https://books. google.com/books?hl=es\&lr=lang_en\&id=G3TYBu6-4G0C\&oi=fnd\&pg=PT $17 \& d q=$ The + Theory+of + Social+and+Economic+Organization+\&ots $=\mathrm{XqOeY}$ MlBH0\&sig=01YIV4V0sTgnO8MYc2eFvKP1DU8 (recuperado el 9-I-2017).

Weinstein, J. (1999). Hate speech, pornography, and the radical attack on free speech doctrine. Boulder: Westview Press.

Wellington, H. (1979). On freedom of expression. The Yale Law Journal, 88(6), 1105-1142. 